\title{
PRISE EN CHARGE TARDIVE DES SEQUELLES DE FRACTURES DU PLANCHER ORBITAIRE
}

\author{
M.A. EL AFRIT, H. KAMMOUN, H. MAZLOUT, S. TROJET, M. HAMDOUNI, A. KRAIEM. \\ SERVICE D'OPHTALMOLOGIE HÔPITAL HABIB THAMEUR TUNIS
}

\begin{abstract}
Introduction : Les fractures du plancher orbitaire, sont devenues une pathologie assez fréquente étant donné, l'augmentation du nombre d'accidents de la route. Le plancher orbitaire de part sa constitution anatomique est certainement le maillon faible du cadre orbitaire.

Observation : Nous présentons le cas d'un jeune de 35 ans polytraumatisé avec des séquelles de fracture du plancher orbitaire qui ne sera opéré que deux années plus tard, priorité chirurgicale oblige ( le rachis étant opéré en premier) avec les risques certains d'échec de la chirurgie ophtalmologique.

Discussion : Les séquelles de ce type de fracture sont exposées, ainsi que les différentes modalités thérapeutiques. L'enophtalmie, la diplopie, les anomalies osseuses et palpébrales constituent autant de séquelles parfois difficiles à prendre en charge.

Les voies d'abord chirurgical sont discutées ; l'usage de matériaux tels les implants en hydroxyapatite également.

Conclusion : Le traitement des fractures du plancher de l'orbite est actuellement bien codifié et doit toujours être tenté même des années après le traumatisme causal.
\end{abstract}

Mots clés : fracture plancher orbitaire, diplopie

\section{SUMMARY}

Introduction : Fractures of the orbit floor are frequent because of the increase of the incidence of road accidents. Du to its constitution, the orbit floor is vulnerable.

Case report : A 35 years old man victim of a road accident who presented 2 years later with after effects of a fracture of the orbit floor. After the traumatism, he had other injuries theatening his life and requiring urgent treatment.

Discussion : We present sequellae of fractures of the orbit floor with different therapeutic modalities.

Enophtalmia, diplopia, bone and palpebral anomalies are often difficult to manage.

Conclusion : Treatment of the fractures of the orbit floor is at present well-codified and should be attempted even several years after the trauma.

Key-words : orbit floor,fracture-diplopia

\section{INTRODUCTION}

Les fractures du plancher de l'orbite voient chaque année leur nombre augmenter, proportionnellement aux nombres d'agressions et d'accidents de la voie publique. De nos jours, les séquelles de ces traumatismes prennent de plus en plus d'importance car les conditions d'exercice de la vie moderne exigent une vision binoculaire et une cinétique oculaire parfaites.

Les fractures isolées du plancher orbitaire par «blow-out» sont volontiers méconnues ou décelées seulement plusieurs semaines après l'accident, la traduction clinique étant essentiellement la diplopie post traumatique, plus tardivement l'enophtalmie.

Cette méconnaissance s'explique par l'absence de troubles morphologiques, par le masquage de la diplopie et de l'enophtalmie au début par l'oedème des paupières, par les examens radiologiques standards qui ne montrent pas de fracture du plancher puisque le cadre orbitaire est intact, enfin par la fréquence avec laquelle on s'abstient de faire une duction forcée.

Nous présentons la prise en charge d'un patient de 35 ans ayant présenté 2 ans auparavant une fracture du plancher orbitaire gauche par «blow-out » dans le cadre d'un accident de la voie publique.

\section{OBSERVATIONS}

II s'agit d'un patient âgé de 35 ans ; aux antécédents d'accident de la voie publique survenu le 24/10/2002, il a été opéré du rachis cervical le 19/05/2003 des suites de son accident.

Le patient a consulté en ophtalmologie en 2004 pour une diplopie, une enophtalmie de l'œil gauche et une rétraction de la paupière supérieure gauche.

L'examen ophtalmologique a trouvé une enophtalmie gauche, une diplopie en regard primaire avec une rétraction de la paupière supérieure gauche. Nous avons également noté l'absence de synergie oculo-palpébrale dans le regard vers le bas du côté gauche. 
L'examen des segments antérieur et postérieur était normal. Un bilan thyroïdien a été demandé, il était normal. Le test de Lancaster a confirmé la limitation de l'élévation (fig 1).

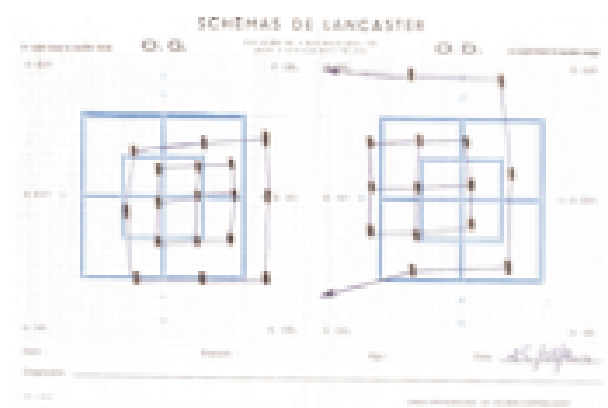

Fig.1 : Le test de Lancaster confirme la limitation d'élévation

Une tomodensitométrie (TDM) orbitaire faite le 20/01/2004 a trouvé une fracture discrètement déplacée du plancher de l'orbite gauche externe latéralisée à gauche.

Devant la gêne occasionnée par la diplopie, l'indication opératoire a été portée malgré l'important taux d'échec rapporté dans la littérature à distance du traumatisme. Le patient a été opéré le 27/07/2004 (fig. 2).

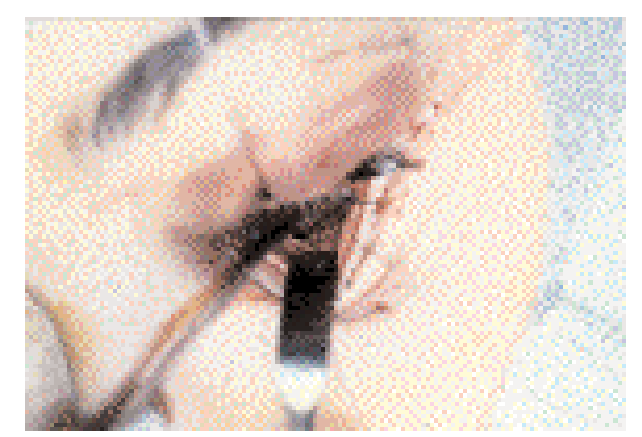

Fig.2 : Voie d'abord transconjonctivale palpébrale.

L'intervention a comporté les temps suivants : un abord trans-conjonctival jusqu'au périoste et travail sous périosté, un écartement des tissus périorbitaires par lame malléable, un comblement du defect osseux par un tissu périorbitaire fibreux, un dégagement doux de la fibrose et mise en place du greffon de silicone.

Le résultat post-opératoire était une disparition de la limitation d'élévation et de l'enophtalmie (fig 3, 4).

Un TDM de contrôle à 2 mois post-opératoire a trouvé (fig 5) : un matériel d'ostéosynthèse en place et une absence d'incarcération musculaire.

Le patient a quant même été repris chirurgicalement vue la persistance d'une rétraction de la paupière supérieure gauche le 27/01/2005 : il a bénéficié d'une myotomie du Müller par voie conjonctivale (fig 6).

L'examen à 7 post-opératoire a trouvé une disparition de la rétraction palpébrale supérieure gauche. On a assisté à une persistance d'une diplopie uniquement dans le regard en haut.
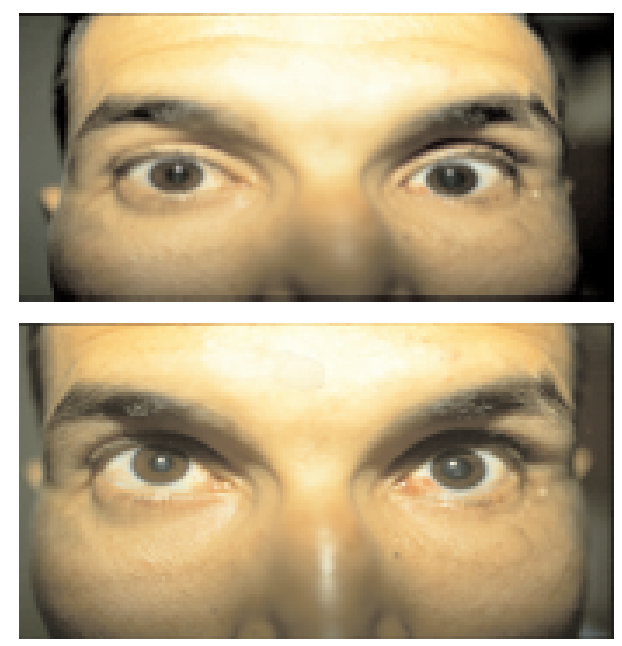

Fig.3 et 4 : Le résultat post-opératoire montre la disparition de la limitation d'élévation et de l'enophtalmie
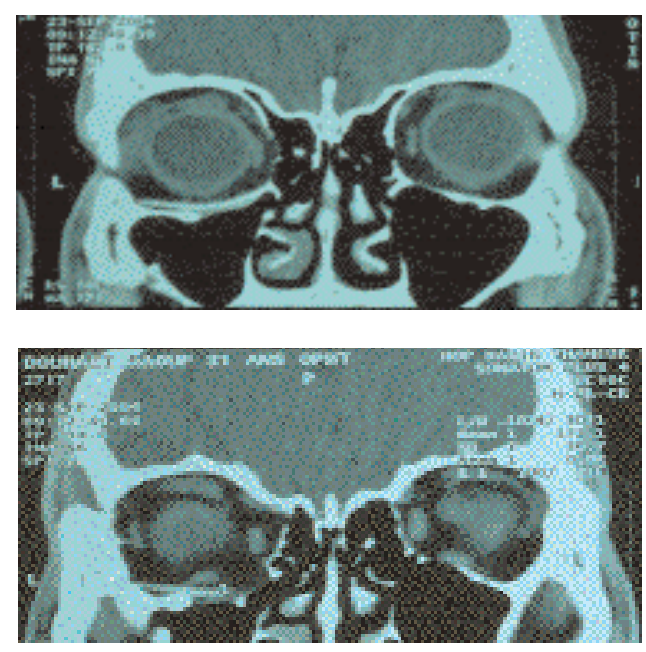

Fig.5 : TDM post-opératoire : absence d'incarcération musculaire

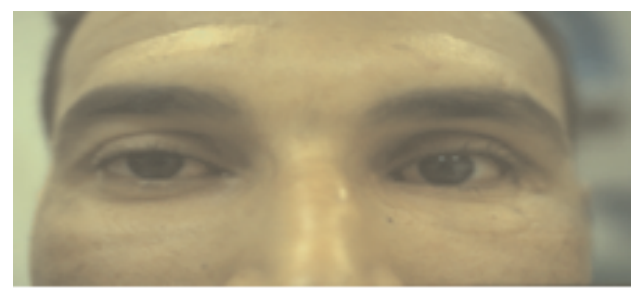

Fig.6 : Disparition de la rétraction de la paupière supérieure après myotomie de Muller 


\section{DISCUSSION}

Les fractures du plancher orbitaire par «blow-out» correspondent aux traumatismes avec déplacement externe des murs orbitaires. Elles peuvent être pures ou impures selon l'intégrité des rebords orbitaires.

Les séquelles des fractures «blow out » restent nombreuses en raison de la méconnaissance des lésions en urgence ou d'une prise en charge thérapeutique inadaptée. Elles consistent en :

- une baisse visuelle ou cécité : ce n'est pas le cas pour notre patient dont le globe est intact.

- une diplopie : c'est une des principales complications qui fait l'objet de plaintes répétées des patients. Elle est d'autant plus invalidante qu'elle se manifeste dans le regard primaire et à la lecture. La confrontation de l'examen clinique, du test de Lancaster, de l'imagerie, et du test de duction forcée, permet de préciser le mécanisme du trouble oculomoteur $(4,6)$.

- une énophtalmie : Le plus souvent tardive, elle est liée à une modification des rapports contenant osseux et contenu orbitaire $(3,5)$. Elle s'explique non seulement par le défect osseux, mais aussi par l'existence d'une brèche dans la périorbite, et par une lésion du système ligamentaire suspenseur du globe. L'enophtalmie post-traumatique s'apprécie cliniquement en se référant surtout à la forme de la fente palpébrale qui est dans ces cas rétrécie; chez notre patient elle est plutôt élargie par les rétractions palpébrales inférieures et surtout supérieures.

- des troubles de la sensibilité du nerf sous orbitaire : dans la majorité des cas, l'anesthésie sous orbitaire régresse rapidement. La gêne séquellaire peut se présenter sous forme de paresthésies. En cas de névralgies rebelles, une décompression chirurgicale du canal infra orbitaire ou dans les formes rebelles une neurotomie du nerf maxillaire supérieur peuvent être discutées ; nous n'avons pas eu de troubles de la sensibilité chez ce patient.

- des anomalies osseuses : elles sont très variées depuis le simple décalage des rebords orbitaires jusqu'au déplacement global de l'orbite avec dystopie osseuse. Ces anomalies osseuses s'accompagnent souvent de déformations des parties molles à type de cicatrices vicieuses, de décalage des repères comme le sourcil, la commissure palpébrale, les canthi interne ou externe, d'un trouble de la position oculaire et bien souvent d'un désordre oculomoteur.

Nous n'avons pas eu de séquelles morphologiques chez ce patient.

- des séquelles palpébrales : un ptôsis post traumatique peut être lié soit à l'enophtalmie, soit à une lésion neurologique par paralysie de la troisième paire crânienne, soit à une atteinte du muscle releveur lui même.

Souvent une chirurgie oculomotrice secondaire est nécessaire ; dans notre cas on pourrait prévoir simplement un recul du droit supérieur controlatéral.

Le traitement des traumatismes orbitaires doit intégrer les troubles fonctionnels et les lésions anatomiques du contenu orbitaire engendrées par le déplacement du squelette osseux.

C'est seulement à ce prix que l'intégrité fonctionnelle et esthétique sera restaurée.

En dehors d'une prise en charge urgente et prioritaire d'une plaie du globe, le traitement des fractures « blowout 》 obéit à plusieurs grands principes : une large exposition du foyer de fracture à l'aide d'une ou plusieurs voies d'abord adaptées, libération du foyer de fracture de la péri orbite et des ailerons musculaires, réduction des fragments osseux déplacés et contention par ostéosynthèse au fil d'acier ou par plaques vissées $(7,8,9)$, comblement des défects osseux par greffes osseuses ou implants, et correction des lésions des parties molles (oculomotrices, palpébrales canthales ou lacrymales) en dernier lieu.

Les voies d'abords chirurgicales pour le plancher orbitaire sont essentiellement la voie transconjonctivale palpébrale qui est indiquée dans les petites fractures du plancher assez antérieures et sans grand déplacement $(1,2)$. La voie cutanée palpébrale inférieure qu'elle soit sous ciliaire, infratarsale ou en regard du rebord infra-orbitaire offre un large jour sur le plancher, le rebord inférieur et au besoin la paroi médiane de l'orbite.

Le comblement des défects osseux peut nécessiter soit une autogreffe osseuse soit des implants allogènes.

L'autogreffe osseuse, prélevée sur le patient lui même (crête iliaque, os pariétal, gril costal), constitue la méthode de choix. Elle est cependant réservée aux défects majeurs ou pour la correction de l'enophtalmie au stade de séquelles $(10,11)$.

Les implants allogènes sont largement employés évitant les inconvénients du prélèvement osseux. On dispose, à I'heure actuelle des implants métalliques à type de treillis de vitallium ou de titanium qui offrent un support rigide, fin et malléable, des implants à résorption spontanée servant de tuteur à un cale ostéofibreux (treillis de Vicryl, PDS, hydroxy apatite, corail) ou des implants non résorbables en silicone, supramid ou en téflon (nous avons utilisés du silicone).

L'épaisseur de l'implant varie suivant l'importance du défect osseux et dans tous les cas ne dépassera pas les $2 \mathrm{~mm}$ pour réduire les complications iatrogènes (hypotropie du globe, compression, migration ou extrusion de l'implant). L'important est que le greffon trouve un appui périphérique sur l'os sain. Dans les fractures du plancher, la position de l'implant est telle que son bord antérieur atteint le rebord orbitaire et que son bord postérieur ne menace pas l'apex orbitaire. La fixation de l'implant est parfois nécessaire, réalisée soit par suture périostée soit par des fils d'acier ou des mini-plaques.

L'indication opératoire d'une fracture isolée du plancher repose sur la présence d'au moins un des critères suivants : persistance d'une diplopie significative d'origine 
mécanique avec test de duction forcée positif dans le regard primaire ou en position de lecture, passé un délai de quinze jours. Celle-ci demeure la principale indication thérapeutique; présence d'une enophtalmie supérieure à $2 \mathrm{~mm}$ responsable de séquelles esthétiques majeures, plus particulièrement en cas de dystopie oculaire associée ; présence d'un volumineux déficit osseux (supérieur à $50 \%$ de la surface du plancher) ou d'un gros délabrement inférieur du plancher dans la cavité sinusienne à l'origine d'une expansion du volume orbitaire (supérieur à $25 \%$ ) responsable d'une enophtalmie secondaire;

D'autres séquelles peuvent également mener à la chirurgie telles que : la ptose du globe oculaire ou l'hypoesthésie du nerf sous orbitaire. Cette dernière est source de controverse. Elle n'est pas une indication opératoire formelle. Elle est en général due à une compression ou un oedème, et régresse le plus souvent spontanément en trois à six mois.

Enfin, retenons que la règle est d'opérer dans les quinze jours suivant le traumatisme lorsque l'oedème et l'hématome auront disparu et surtout lorsque la diplopie aura fait la preuve de sa permanence et de son origine mécanique.

\section{CONCLUSION}

Les fractures du plancher de l'orbite exposent à des séquelles tant fonctionnelles qu'esthétiques très invalidantes.

Le succès thérapeutique repose sur un diagnostic et un traitement précoces.

Le but du traitement est la restauration de l'orbite mais également la cure des déséquilibres oculomoteurs découlant de la fracture, afin d'en éviter les séquelles très invalidantes dans la vie moderne.

Mais il ne faut pas hésiter à opérer ; même tardivement des résultats spectaculaires peuvent être obtenus.

\section{REFERENCES}

1. Ceccarini M. Fractures isolées du plancher de l'orbite. Aspects cliniques et médico légaux. Thèse Montpellier, 1978.

2. Corbeilles R. Les séquelles des fractures du plancher de l'orbite. Thèse Lyon, 1974.

3. Converse JM, Smith B. Enophtalmos and diplopia in fractures of the orbital floor Birt J Plast Surg 1957; 9 (4):265 74.

4. Defay JP Les fractures du plancher de l'orbite et la récupération juridique. Thèse Montpellier, 1987.

5. Seiff SR, Good WV. Hypertropia and the posterior blowout fracture: Mechanism and management. Ophthlamology 1996; 103 (1):152 6.

6. Daieff CY. Une association mal comprise : diplopie et plancher de l'orbite. Ann Chir Plast 1969; 14 (2):133 6.
7. Stewart MG, Patrinely JR, Appling WD, Jordan DR, Late proptosis following orbital floor fracture repair. Arch Otorhinolaryngol Head Neck Surg 1995; 121 (6):649 52.

8. Sparfel 0, Raybaud 0, Potard G, Guibal Y, et al. Reconstruction des fractures du plancher de l'orbite. J Fr ORL 1997; 46 (3).

9. Yab K, Tajima S, Ohba S. Displacements of eyeball in orbital blow out fractures. Plast Recons Surg 1997; 100 (6):1409 17.

10. Morax S, Pascal D. Traitement chirurgical des diplopies secondaires à une fracture du plancher de l'orbite. J Fr Ophtalmol 1984; 7 (10):633 47.

11. Biesman BS, Hornblass A, Lisman R, Kazlas M. Diplopia after surgical repair of orbital floor fractures. Ophthal Plast Reconstr Surg 1996; 12 (1):9 16. 